\title{
Erratum to: Universities between traditional forces and modern demands: the role of imprinting on the missions of German universities
}

\author{
Simon Oertel ${ }^{1} \cdot$ Matthias Söll $^{2}$
}

\section{Erratum to: High Educ DOI 10.1007/s10734-016-0013-0}

Unfortunately, there was a compositor's error (format error) in Tables 2 and 3 in the original publication. The corrected tables have been given below.

Tables 2 and 3 have also been corrected in the original article.

The online version of the original article can be found under doi:10.1007/s10734-016-0013-0.

\section{Simon Oertel}

simon.oertel@uni-jena.de

1 Chair of Organization, Leadership, and Human Resource Management, School of Economics and Business Administration, Friedrich Schiller University Jena, Carl-Zeiss-Strasse 3, 07743 Jena, Germany

2 Chair of Business and Economics Didactics, Institute of Vocational Education, Faculty of Economics and Management, University of Kassel, Heinrich-Plett-Strasse 40, 34132 Kassel, Germany 
Table 2 Effects on the likelihood of having a mission statement

\begin{tabular}{|c|c|c|}
\hline & $\begin{array}{l}(1) \\
\text { Mission statement } \\
(1=\text { yes; } 0=\text { no })\end{array}$ & $\begin{array}{l}(2) \\
\text { Mission statement } \\
(1=\text { yes; } 0=\text { no })\end{array}$ \\
\hline Founded in pre-classic period (before 1808) & & $\begin{array}{l}0.644 \\
(1.641)\end{array}$ \\
\hline Founded in classic period (1809-1960) & & $\begin{array}{l}-0.212 \\
(0.769)\end{array}$ \\
\hline Power & $\begin{array}{l}1.066 \\
(2.395)\end{array}$ & $\begin{array}{l}1.154 \\
(2.501)\end{array}$ \\
\hline Reputation & $\begin{array}{c}-0.093 \\
(0.059)\end{array}$ & $\begin{array}{c}-0.094 \\
(0.060)\end{array}$ \\
\hline Age/100 & $\begin{array}{l}0.603 \\
(0.565)\end{array}$ & $\begin{array}{l}0.328 \\
(1.116)\end{array}$ \\
\hline$(\text { Age } / 100)^{2}$ & $\begin{array}{c}-0.102 \\
(0.100)\end{array}$ & $\begin{array}{c}-0.080 \\
(0.148)\end{array}$ \\
\hline Size/1000 & $\begin{array}{c}-0.010 \\
(0.028)\end{array}$ & $\begin{array}{c}-0.009 \\
(0.028)\end{array}$ \\
\hline Internationality & $\begin{array}{l}3.669 \\
(2.420)\end{array}$ & $\begin{array}{l}3.704 \\
(2.516)\end{array}$ \\
\hline Business department & $\begin{array}{c}-0.414 \\
(0.539)\end{array}$ & $\begin{array}{c}-0.504 \\
(0.537)\end{array}$ \\
\hline Private university & $\begin{array}{l}1.813 * \\
(1.006)\end{array}$ & $\begin{array}{l}1.768 * \\
(1.002)\end{array}$ \\
\hline Third-party funding/100,000 & $\begin{array}{l}1.327 \\
(1.396)\end{array}$ & $\begin{array}{l}1.533 \\
(1.403)\end{array}$ \\
\hline University of technology & $\begin{array}{l}-0.727 \\
(0.748)\end{array}$ & $\begin{array}{l}-0.843 \\
(0.765)\end{array}$ \\
\hline Visibility (in Mio. hits) & $\begin{array}{l}3.468 * * \\
(1.524)\end{array}$ & $\begin{array}{l}3.321 * * \\
(1.529)\end{array}$ \\
\hline Constant & $\begin{array}{c}-0.830 \\
(0.597)\end{array}$ & $\begin{array}{l}-0.636 \\
(0.656)\end{array}$ \\
\hline$R^{2}$ & 0.13 & 0.14 \\
\hline Log-likelihood & -61.58 & -61.33 \\
\hline Observations & 112 & 112 \\
\hline
\end{tabular}

Reference category: founded in modern period (after 1961)

Robust standard errors in parentheses

$* * * p<0.01$; ** $p<0.05 ; * p<0.1$ 
Table 3 Effects on the proportion of institutional service-logic contents in mission statements of universities

\begin{tabular}{|c|c|c|c|c|}
\hline & $\begin{array}{l}(3) \\
\text { Institutional } \\
\text { logic } \\
(1=100 \% \\
\text { service-logic })\end{array}$ & $\begin{array}{l}(4) \\
\text { Institutional } \\
\text { logic } \\
(1=100 \% \\
\text { service-logic })\end{array}$ & $\begin{array}{l}(5) \\
\text { Institutional } \\
\text { logic } \\
(1=100 \% \\
\text { service-logic })\end{array}$ & $\begin{array}{l}(6) \\
\text { Institutional } \\
\text { logic } \\
(1=100 \% \\
\text { service-logic })\end{array}$ \\
\hline $\begin{array}{l}\text { Founded in pre-classic period (before } \\
\text { 1808) }\end{array}$ & & $\begin{array}{l}-0.871 * * \\
(0.444)\end{array}$ & $\begin{array}{l}-0.686 \\
(0.453)\end{array}$ & $\begin{array}{l}-0.734 * \\
(0.408)\end{array}$ \\
\hline Founded in classic period (1809-1960) & & $\begin{array}{l}-0.105 \\
(0.266)\end{array}$ & $\begin{array}{l}-0.009 \\
(0.331)\end{array}$ & $\begin{array}{l}-0.277 \\
(0.305)\end{array}$ \\
\hline $\begin{array}{l}\text { Founded in pre-classic period (before } \\
1808) * \text { power }\end{array}$ & & & $\begin{array}{l}-4.460 * \\
(2.345)\end{array}$ & \\
\hline $\begin{array}{l}\text { Founded in classic period (1809-1960) } \\
* \text { power }\end{array}$ & & & $\begin{array}{l}-1.321 \\
(1.841)\end{array}$ & \\
\hline $\begin{array}{l}\text { Founded in pre-classic period (before } \\
1808) * \text { reputation }\end{array}$ & & & & $\begin{array}{l}0.024 \\
(0.029)\end{array}$ \\
\hline $\begin{array}{l}\text { Founded in classic period (1809-1960) } \\
* \text { reputation }\end{array}$ & & & & $\begin{array}{l}0.055^{* *} * \\
(0.027)\end{array}$ \\
\hline Power & $\begin{array}{l}1.308 * \\
(0.743)\end{array}$ & $\begin{array}{l}1.288 \\
(0.788)\end{array}$ & $\begin{array}{l}2.590 \\
(1.943)\end{array}$ & $\begin{array}{l}1.181 * \\
(0.713)\end{array}$ \\
\hline Reputation & $\begin{array}{l}0.008 \\
(0.017)\end{array}$ & $\begin{array}{l}0.010 \\
(0.015)\end{array}$ & $\begin{array}{l}0.006 \\
(0.015)\end{array}$ & $\begin{array}{l}-0.023 \\
(0.026)\end{array}$ \\
\hline Age/100 & $\begin{array}{l}-0.057 \\
(0.180)\end{array}$ & $\begin{array}{l}0.353 \\
(0.250)\end{array}$ & $\begin{array}{l}0.354 \\
(0.265)\end{array}$ & $\begin{array}{l}0.218 \\
(0.251)\end{array}$ \\
\hline$(\text { Age } / 100)^{2}$ & $\begin{array}{l}0.002 \\
(0.032)\end{array}$ & $\begin{array}{l}-0.037 \\
(0.035)\end{array}$ & $\begin{array}{l}-0.021 \\
(0.035)\end{array}$ & $\begin{array}{l}-0.020 \\
(0.039)\end{array}$ \\
\hline Size/1000 & $\begin{array}{l}-0.012 * * \\
(0.006)\end{array}$ & $\begin{array}{l}-0.015^{* *} \\
(0.006)\end{array}$ & $\begin{array}{l}-0.014 * * \\
(0.006)\end{array}$ & $\begin{array}{l}-0.015^{* * *} \\
(0.005)\end{array}$ \\
\hline Internationality & $\begin{array}{l}-0.469 \\
(0.466)\end{array}$ & $\begin{array}{l}-0.490 \\
(0.478)\end{array}$ & $\begin{array}{l}-0.607 \\
(0.508)\end{array}$ & $\begin{array}{l}-0.365 \\
(0.475)\end{array}$ \\
\hline Business department & $\begin{array}{l}0.051 \\
(0.193)\end{array}$ & $\begin{array}{l}0.102 \\
(0.191)\end{array}$ & $\begin{array}{l}0.054 \\
(0.198)\end{array}$ & $\begin{array}{l}0.174 \\
(0.193)\end{array}$ \\
\hline Private university & $\begin{array}{l}-0.010 \\
(0.258)\end{array}$ & $\begin{array}{l}-0.019 \\
(0.252)\end{array}$ & $\begin{array}{l}0.095 \\
(0.262)\end{array}$ & $\begin{array}{l}-0.173 \\
(0.280)\end{array}$ \\
\hline Third-party funding/100,000 & $\begin{array}{l}-0.096 \\
(0.316)\end{array}$ & $\begin{array}{l}-0.185 \\
(0.301)\end{array}$ & $\begin{array}{l}-0.198 \\
(0.301)\end{array}$ & $\begin{array}{l}-0.099 \\
(0.295)\end{array}$ \\
\hline University of technology & $\begin{array}{l}0.130 \\
(0.187)\end{array}$ & $\begin{array}{l}0.159 \\
(0.168)\end{array}$ & $\begin{array}{l}0.187 \\
(0.170)\end{array}$ & $\begin{array}{l}-0.051 \\
(0.199)\end{array}$ \\
\hline Visibility (in Mio. hits) & $\begin{array}{l}0.144 \\
(0.096)\end{array}$ & $\begin{array}{l}0.110 \\
(0.073)\end{array}$ & $\begin{array}{l}0.106 \\
(0.080)\end{array}$ & $\begin{array}{l}0.051 \\
(0.078)\end{array}$ \\
\hline Constant & $\begin{array}{l}-0.369 \\
(1.175)\end{array}$ & $\begin{array}{l}0.695 \\
(1.402)\end{array}$ & $\begin{array}{l}1.125 \\
(1.322)\end{array}$ & $\begin{array}{l}0.947 \\
(1.381)\end{array}$ \\
\hline Log-likelihood & -35.38 & -35.23 & -34.99 & -35.07 \\
\hline AIC & 1.26 & 1.31 & 1.36 & 1.36 \\
\hline Observations & 75 & 75 & 75 & 75 \\
\hline
\end{tabular}

Reference category: founded in modern period (after 1961)

Robust standard errors in parentheses

*** $p<0.01$; ** $p<0.05$; * $p<0.1$ 